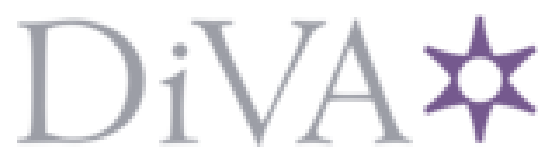

http://www.diva-portal.org

This is the published version of a paper published in Journal of Chemical Physics.

Citation for the original published paper (version of record):

Eland, J H., Zagorodskikh, S., Squibb, R J., Mucke, M., Sorensen, S L. et al. (2014)

Carbon dioxide ion dissociations after inner shell excitation and ionization: The origin of sitespecific effects.

Journal of Chemical Physics, 140(18): 184305

http://dx.doi.org/10.1063/1.4872218

Access to the published version may require subscription.

N.B. When citing this work, cite the original published paper.

Permanent link to this version:

http://urn.kb.se/resolve?urn=urn:nbn:se:uu:diva-228478 


\title{
Carbon dioxide ion dissociations after inner shell excitation and ionization: The origin of site-specific effects
}

\author{
J. H. D. Eland, ${ }^{1,2}$ S. Zagorodskikh, ${ }^{2}$ R. J. Squibb, ${ }^{2,3}$ M. Mucke, ${ }^{2}$ S. L. Sorensen, ${ }^{4}$ \\ and R. Feifel ${ }^{2,3, a)}$ \\ ${ }^{1}$ Department of Chemistry, Physical and Theoretical Chemistry Laboratory, Oxford University, \\ South Parks Road, Oxford OX1 3QZ, United Kingdom \\ ${ }^{2}$ Department of Physics and Astronomy, Uppsala University, 75120 Uppsala, Sweden \\ ${ }^{3}$ Department of Physics, Gothenburg University, 41296 Gothenburg, Sweden \\ ${ }^{4}$ Division of Synchrotron Radiation Research, Lund University, 22100 Lund, Sweden
}

(Received 7 February 2014; accepted 10 April 2014; published online 9 May 2014)

\begin{abstract}
Multi-coincidence experiments with detection of both electrons and ions from decay of core-excited and core-ionized states of $\mathrm{CO}_{2}$ confirm that $\mathrm{O}_{2}{ }^{+}$is formed specifically in Auger decay from the $\mathrm{C} 1 \mathrm{~s}-\pi *$ and $\mathrm{O} 1 \mathrm{~s}-\pi *$ resonances. Molecular rearrangement occurs by bending in the resonant states, and $\mathrm{O}_{2}{ }^{+}$is produced by both single and double Auger decay. It is suggested that electron capture by $\mathrm{C}^{+}$after partial dissociation in the doubly ionized core of excited $\mathrm{CO}_{2}{ }^{+}$, formed by shake-up in spectator resonant Auger decay, accounts for high kinetic energy and high internal energy in some $\mathrm{C}$ $+\mathrm{O}_{2}{ }^{+}$fragments. (C) 2014 AIP Publishing LLC. [http://dx.doi.org/10.1063/1.4872218]
\end{abstract}

\section{INTRODUCTION}

The dissociations of positive ions derived from $\mathrm{CO}_{2}$ have been extensively studied both experimentally ${ }^{1-20}$ and theoretically. ${ }^{21-24}$ Attention has recently concentrated particularly on the formation and dissociations of the doubly $5,7,10$ and triply ${ }^{11,12,25}$ charged ions from $\mathrm{CO}_{2}$, particularly those formed by high energy photon impact. In such a triatomic neutral or singly ionized molecule the density of states is so low that complete randomization of internal energy is not to be expected, and the characteristics of its dissociation depend not only on the total internal energy, but also on the precise way in which it is distributed between quantum states. For highly excited or highly charged ions, although the total internal energy and the density of electronic states can be very much higher, some state- and site-specific behavior may still be expected. In a recent paper Laksman et al. ${ }^{13}$ show that ionization of $\mathrm{CO}_{2}$ by initial excitation of a $1 \mathrm{~s}$ electron from either the $\mathrm{C}$ or the $\mathrm{O}$ atom to a $\pi^{*}$ orbital, thus creating a highly excited state, results in formation of $\mathrm{O}_{2}{ }^{+}$ions, presumably by strong bending of the molecule. This reaction is not observed after ionization of $\mathrm{CO}_{2}$ by other pathways; for comparison formation of $\mathrm{S}_{2}{ }^{+}$occurs in valence ionization of $\mathrm{CS}_{2},{ }^{26,27}$ but $\mathrm{SO}^{+}$is not detected from OCS valence ionization. ${ }^{28}$ Laksman et al. ${ }^{13}$ also show by ion-ion correlation that at least some of the $\mathrm{O}_{2}{ }^{+}$arises from double ionization, as it is detected in coincidence with $\mathrm{C}^{+}$ions. They suggest that this may be its only formation pathway. In the present work, we test this suggestion by adding electron detection in a multi-particle coincidence study.

In a related earlier work, Öhrwall et al. ${ }^{7}$ showed that long-lived $\mathrm{CO}_{2}{ }^{++}$ions are less abundant in Auger decay from a $\mathrm{C} 1 \mathrm{~s}$ hole state of $\mathrm{CO}_{2}$ than from an $\mathrm{O} 1 \mathrm{~s}$ hole state. This

\footnotetext{
a) Author to whom correspondence should be addressed. Electronic mail: raimund.feifel@physics.gu.se
}

could be due either to different behavior of specific states populated in the two Auger decays, or to different energy deposition functions in the two cases, the states concerned reacting in the same way once populated. Shibata et al. ${ }^{29}$ have now shown by examination of the Auger electron spectra at high resolution that the latter explanation is the more probable, as decay from an $\mathrm{O} \mathrm{K}$-hole gives a higher proportion of high energy Auger electrons (low $\mathrm{CO}_{2}{ }^{++}$internal energy) than decay from a $\mathrm{C} \mathrm{K}$ hole. This is also consistent with the localization of the $\pi^{*}$ orbital on the $\mathrm{O}$ atom. Both Laksman et al. ${ }^{13}$ and Öhrwall et al. ${ }^{7}$ also observed that $\mathrm{O}_{2}{ }^{+}$is produced less abundantly at the $\mathrm{O} 1 \mathrm{~s}-\pi^{*}$ resonance than at the $\mathrm{C} 1 \mathrm{~s}-\pi^{*}$ resonance. This might again be due either to a difference in the energy deposition function or to different behavior of specific states populated in the respective decays. We also test these hypotheses.

\section{EXPERIMENTAL}

This work was done on beamline U49/2 PGM-1 at the storage ring BESSY-II of the Helmholtz Zentrum, Berlin. It used the multi-electron-ion coincidence magnetic bottle developed in Oxford ${ }^{30,31}$ with minor modifications. Briefly, ionization occurs at the crossing of an effusive jet of target gas and the monochromatised soft $\mathrm{x}$-ray beam from the synchrotron radiation storage ring. Once electrons have left the source region an electric extraction pulse is applied to accelerate all ions through the hollow pole of a permanent magnet where they are accelerated further and traverse a drift space before being registered at an MCP detector. Ion and electron flight times relative to the light pulses passed to the apparatus at $10 \mathrm{kHz}$ by our synchronous chopper ${ }^{32}$ are digitized and stored on a local computer.

Because of the weaker magnetic field from the hollow magnet compared to the usual conical magnet, ${ }^{33}$ the electron energy resolution is about $5 \%$. Mass resolution $M / \Delta M$ for 
thermal ions is about 150 (FWHM) and remains fully adequate to resolve the product ions from $\mathrm{CO}_{2}$ in all charge states, even when they carry significant kinetic energies. The measured collection efficiency for electrons is $35 \%-40 \%$ for energies up to $300 \mathrm{eV}$ and probably slightly lower for higher energies. Because of some unintended electric potentials, electrons of energy below $2 \mathrm{eV}$ are not collected, which has some effect on the apparent collection efficiency, depending on the real energy distribution. The effective collection efficiency for thermal ions is about $20 \%$, but ions formed with high kinetic energies are gathered less efficiently. The losses also depend on such ions' angular distributions and spatial distributions within the source region. For this reason, the results given in this paper on relative yields of different ions are qualitative rather than exact. On the other hand the kinetic energies and other characteristics generally change only slightly with internal energy of the parent species, so the data are comparable among themselves. The energy calibration (conversion from flight time to energy) is also affected by the magnetic field and electrode arrangement. No very simple expression is valid over the whole electron energy range needed and instead a polynomial in $1 /\left(t-t_{0}\right)$, where $t_{0}$ is the common time offset, is fitted by a least squares routine to relate measured times to the known energies of calibration lines.

\section{DATA ANALYSIS}

Data analysis to determine collection efficiencies and hence branching ratios is based on simple equations for the numbers of true coincidences $\mathrm{C}$. In the expressions below, $\mathrm{N}$ is the number of events of the stated type, $\mathrm{f}_{\mathrm{e}}$ is the collection efficiency for electrons and $f_{i}$ is the collection efficiency for ions.

For a process producing one ion and one electron (parent or fragment from single ionization): $\mathrm{C}=\mathrm{Nf}_{\mathrm{i}} \mathrm{f}_{\mathrm{e}}$.

For a process giving one ion and two electrons $\left(\mathrm{p}^{++}\right.$or fragment $^{++}$in double ionization):

If both electrons are detected $\quad \mathrm{C}=\mathrm{N}_{\mathrm{i}} \mathrm{f}_{\mathrm{e}}{ }^{2}$.

If only one electron is detected $\mathrm{C}=2 \mathrm{~N}_{\mathrm{i}} \mathrm{f}_{\mathrm{e}}\left(1-\mathrm{f}_{\mathrm{e}}\right)$.

For a process giving two ions and two electrons (ion-pair formation in DI):

If all particles are detected $\mathrm{C}=\mathrm{N} \mathrm{f}_{\mathrm{i}}{ }^{2} \mathrm{f}_{\mathrm{e}}{ }^{2}$.

If two ions and one electron are detected

$\mathrm{C}=2 \mathrm{~N} \mathrm{f}_{\mathrm{i}}^{2} \mathrm{f}_{\mathrm{e}}\left(1-\mathrm{f}_{\mathrm{e}}\right)$.

If one particular ion and two electrons are detected $\mathrm{C}=\mathrm{N} \mathrm{f}_{\mathrm{e}}^{2} \mathrm{f}_{\mathrm{i}}\left(1-\mathrm{f}_{\mathrm{i}}\right)$.

If one particular ion and one electron is detected

$\mathrm{C}=2 \mathrm{~N} \mathrm{f}_{\mathrm{e}}\left(1-\mathrm{f}_{\mathrm{e}}\right) \mathrm{f}_{\mathrm{i}}\left(1-\mathrm{f}_{\mathrm{i}}\right)$.

If two ions but no electrons are detected

$$
\mathrm{C}=\mathrm{Nf}_{\mathrm{i}}{ }^{2}\left(1-\mathrm{f}_{\mathrm{e}}\right)^{2} \text {. }
$$

Because an individual dissociation process cannot be identified from the electrons alone, the cases where only electrons are detected are of no practical interest here.

The factor of 2 arises when one of two indistinguishable particles is detected, such as one of two ions of identical mass (e.g., $\mathrm{O}^{+}+\mathrm{O}^{+} / \mathrm{CO}_{2}$ ) or one of two electrons from a continuous distribution. Where the electrons are distinguished by en- ergy (e.g., a photoelectron and an Auger electron), the factor 2 is omitted.

These formulae apply strictly to true coincidences. Accidental coincidences must either be subtracted or reduced to a negligible level by use of a low event rate before the formulae are applied. A very low event rate was used in this work. Unwanted real coincidences such as those arising from higher orders of the ionizing light or from secondary processes must also be allowed for if they overlap features of physical interest. As demonstrated in earlier work, ${ }^{30,31}$ detection of electrons in coincidence with photoions eliminates almost all such interferences.

\section{RESULTS}

We made measurements at five photon energies, 95, 290.7, 350, 535.4, and $620 \mathrm{eV}$. At the three lowest energies there are considerable contributions from second-order light, clearly recognisable in the electron spectra. Other important limitations on the results are that the collection efficiencies for both ions and electrons are rather low, and that there are unwanted electrons, mainly at low energy, arising as secondaries from collisions of primary electrons on solid surfaces (magnet, grids, walls).

We focus first on the question posed by Laksman et al. ${ }^{13}$ whose ion-ion coincidence experiment is sensitive only to double (and higher order) ionization processes: is $\mathrm{O}_{2}{ }^{+}$produced from one or both of single or double ionization? As a first approach to this question we examine mass spectra coincident with different electrons. Figure 1 shows mass spectra from single and double ionization at $290.7 \mathrm{eV}$ (on the

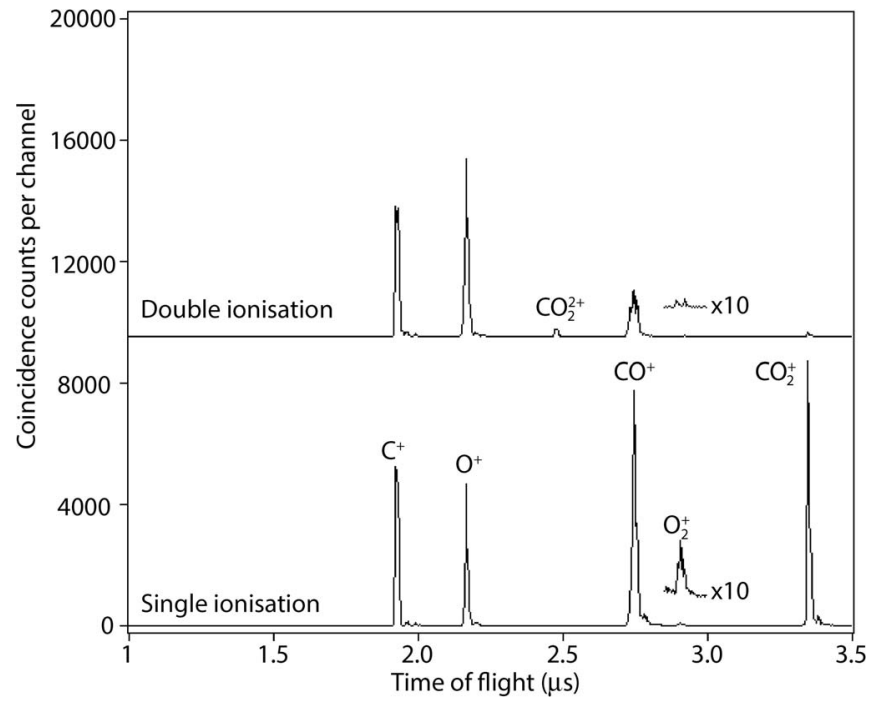

FIG. 1. Mass spectra of $\mathrm{CO}_{2}$ photoionized at the $\mathrm{C} 1 \mathrm{~s}-\pi *$ resonance, $290.7 \mathrm{eV}$. The single ionization spectrum shows ions detected in coincidence with a single electron of energy in the nominal energy range $250 \mathrm{eV}$ to $300 \mathrm{eV}$, while the double ionization spectrum shows ions with electron pairs whose summed energy is in the range 150 to $250 \mathrm{eV}$. Because of the limited energy resolution $(\sim 15 \mathrm{eV}$ at $300 \mathrm{eV})$, these energies are not sharp cut-offs, and because the electron collection efficiency is not $100 \%$, some double ionization is always accounted as single. Furthermore, because of stray background electrons, some single ionization intrudes into the doubles, but the fraction is very small, as can be judged from the intensities of the parent ion. 


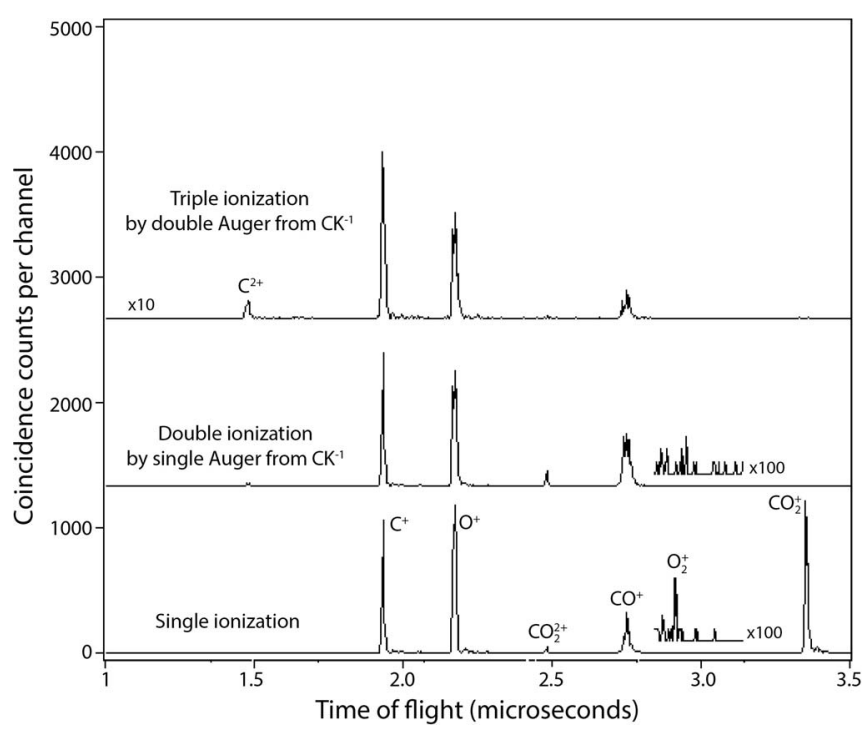

FIG. 2. Mass spectra of $\mathrm{CO}_{2}$ from different stages of ionization at a photon energy of $350 \mathrm{eV}$, above the $\mathrm{C} 1 \mathrm{~s}$ edge. Double and triple ionization events have been selected to include one electron at the energy of the $\mathrm{C} 1 \mathrm{~s}$ photoline. The same limitations apply as in Fig. 1. The common intensity scale applies with displacements to the two lower traces, while the uppermost spectrum has been amplified by a factor of ten.

$\mathrm{C} 1 \mathrm{~s}-\pi *$ resonance), with selection by the number of electrons detected in coincidence ( 1 or 2$)$. A comparable set of mass spectra at $350 \mathrm{eV}$ (above the $\mathrm{C} 1 \mathrm{~s}$ edge and above the related core-valence double ionization energies) is shown in Fig. 2 for comparison. The limited purity of the spectra is explained in the captions.

From Fig. 1 we see that $\mathrm{O}_{2}{ }^{+}$ions are apparently more abundant in single ionization than in double ionization, and that the shape of the $\mathrm{O}_{2}{ }^{+}$peak is different in the two spectra. In double ionization a double peak is seen, consistent with formation of ions with high kinetic energy and the loss of ions which set off at right angles to the spectrometer axis. In the single ionization spectrum, which contains some double ionization because of the imperfect electron collection efficiency, the $\mathrm{O}_{2}{ }^{+}$peak is equally broad but also has a central peak, indicative of low energy ions. The combined observations on intensity and kinetic energy clearly indicate that some $\mathrm{O}_{2}{ }^{+}$ is formed by single ionization. From the form of the peak it seems that some $\mathrm{O}_{2}{ }^{+}$detected in single ionization is nevertheless formed with essentially the same initial kinetic energy as in double ionization. This could happen if in single ionization (simple resonant Auger) the active $\pi^{*}$ electron is shaken up to a higher orbital from which it can be recaptured by one of the ions departing under Coulomb repulsion within the doubly ionized core. Such shake-up processes are well known in "spectator" resonant Auger decay of small molecules. ${ }^{34,35}$ Figure 2 confirms that no $\mathrm{O}_{2}{ }^{+}$at all is formed by single or double Auger decay from the $\mathrm{C} 1 \mathrm{~s}$ core hole, and only a trace, with low kinetic energy, is seen in valence single ionization at $350 \mathrm{eV}$. The possibility that this signal is due to contamination by background air cannot be ruled out. The contrast between double and triple ionization spectra shows that the $\mathrm{C}^{2+}$ ion is formed mainly by triple ionization (double Auger decay).

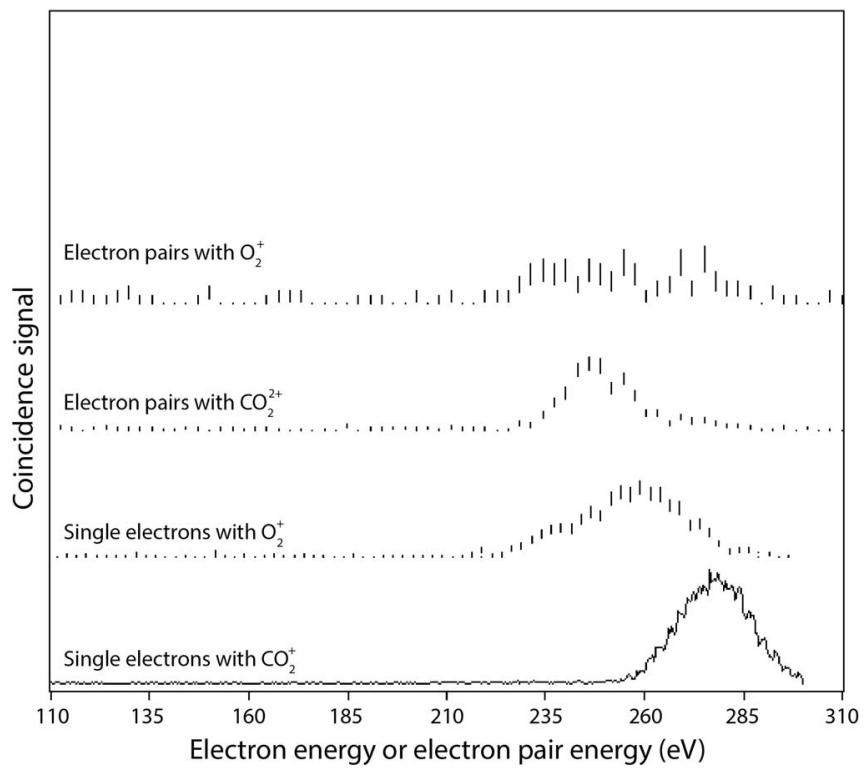

FIG. 3. Energy spectra of single electrons and electron pairs from $\mathrm{CO}_{2}$ after photoionization at the $\mathrm{C} 1 \mathrm{~s}-\pi *$ resonance in coincidence with the $\mathrm{O}_{2}+$ ion and with singly and doubly charged parent ions. Note that the scale shows electron energies, so ion internal energies run in the opposite sense. The electron pair signal in coincidence with $\mathrm{O}_{2}{ }^{+}$is extremely weak, and we believe that the two high points at 269 and $275 \mathrm{eV}$ are statistical fluctuations. The energy bandwidth is estimated as $15 \mathrm{eV}$, but the observed widths for electrons with parent ions are between 20 and $25 \mathrm{eV}$.

As a second approach to the origin of $\mathrm{O}_{2}{ }^{+}$we examine electron spectra coincident with different ions. Figure 3 shows electron spectra coincident with $\mathrm{O}_{2}{ }^{+}$and with singly and doubly charged parent ions for comparison. The $\mathrm{O}_{2}{ }^{+}$ion is found in coincidence with single electrons at binding energies well below the energy onset of the doubly charged parent ion, and in the range where singly charged $\mathrm{CO}_{2}{ }^{+}$is formed. In coincidence with electron pairs the signal is extremely weak, but the main region of significant intensity overlaps the range where $\mathrm{CO}_{2}{ }^{2+}$ is formed and extends also to lower pair energies, i.e., to higher ion energies.

In a third approach, Fig. 4 displays the coincidence map of fragment ions with single electrons at $290.7 \mathrm{eV}$. Examination of the line in the map for $\mathrm{O}_{2}{ }^{+}$ions shows a broadening of the flight time at lower electron energies (higher ion energies) where double ionization is seen. This is an additional confirmation that at the lower ion energies the molecular oxygen ion is formed in single ionization with low kinetic energy release, but in double ionization at higher energy. The fact that it is there formed with $\mathrm{C}^{+}$partner ions is not in doubt, as it was observed by Laksman et al. ${ }^{13}$ who investigated the dynamics in detail, and it is also confirmed here. But if the explanation given above for the formation of high kinetic energy $\mathrm{O}_{2}{ }^{+}$is correct, then $\mathrm{C}^{+}$(with $\mathrm{O}_{2}$ ) ought to be formed by the same mechanism of electron recapture by $\mathrm{O}_{2}{ }^{+}$rather than by $\mathrm{C}^{+}$.

The second question to be addressed is why the yield of $\mathrm{O}_{2}{ }^{+}$is lower at the $\mathrm{O} 1 \mathrm{~s}-\pi^{*}$ resonance than at the C1s$\pi^{*}$ one. Laksman et al. suggest that the reason is the shorter core-hole lifetime for $\mathrm{O}$ (3.5 fs) than for $\mathrm{C}(6 \mathrm{fs})$, implying that the essential step is deformation of the neutral molecule in the core-excited state before electrons are ejected. On this 


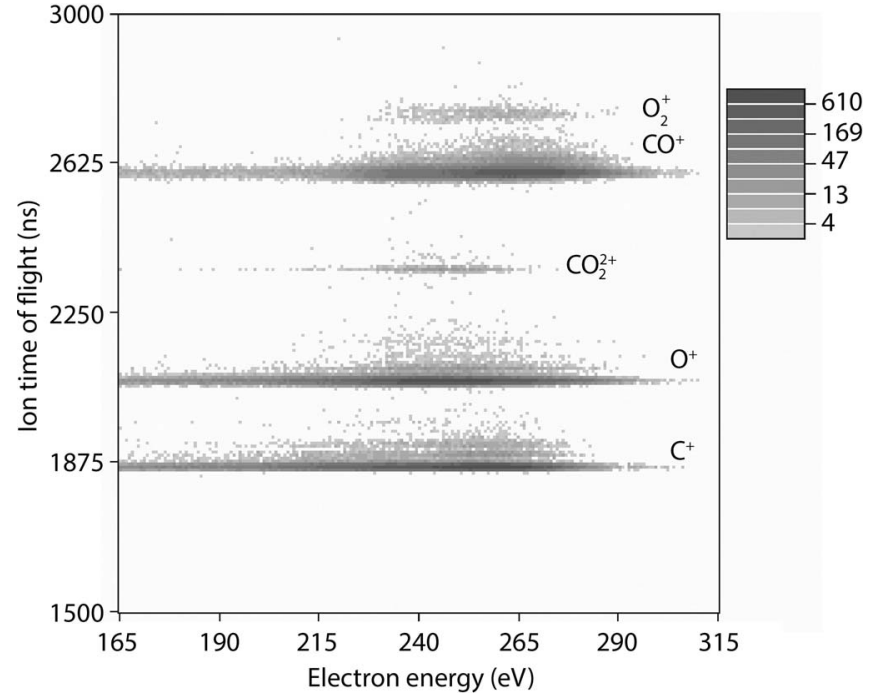

FIG. 4. Coincidence map of fragments detected with one electron after ionization at the $\mathrm{C} 1 \mathrm{~s}-\pi *$ resonance. Because of the low electron collection efficiency, double ionization is seen in the map as well as single. Some water contamination is visible at mass 18 .

interpretation, what happens later is presumably of little importance, and the Auger decay (whether participator or spectator, single or double) must populate states of the nascent ions that dissociate, at least in part, specifically to $\mathrm{O}_{2}{ }^{+}$. In the present measurements, the yield of $\mathrm{C}^{+}+\mathrm{O}_{2}{ }^{+}$is $4 \pm 1$ times less at the $\mathrm{O}$ resonance than at the $\mathrm{C}$ resonance, relative to the main ion pair $\mathrm{O}^{+}+\mathrm{CO}^{+}$. The difference could in principle be due to a difference in the fraction of Auger decays populating some requisite ionized states (the energy deposition) or to different behavior of the particular sets of ionized states populated in the two cases. To test this, we can determine the fragment branching ratios for the same energy in the nascent parent ions, populated from the two resonances. Because of the low resolution (bandwidth $\sim 25 \mathrm{eV}$ at $500 \mathrm{eV}$ ) it would be meaningless to take a much narrower energy range so we have extracted the branching patterns for $20 \mathrm{eV}$ ranges, ion internal energies 30-50 eV and 50-70 eV in single ionization, from the array in Fig. 4 and the equivalent array at $535.4 \mathrm{eV}$. The results are shown in Table I.

At the lower internal energy the branching patterns are almost identical at the two resonances, except for the $\mathrm{O}_{2}{ }^{+}$intensity. At the higher internal energy there is slightly greater difference in the general pattern, but the very strong contrast in

TABLE I. Branching patterns $(\Sigma=1000)$ for the same internal energies of nascent $\mathrm{CO}_{2}{ }^{+}$ions from resonant absorption.

\begin{tabular}{|c|c|c|c|c|}
\hline \multirow[b]{2}{*}{ Internal energy $\mathrm{y}^{\mathrm{a}}$} & \multicolumn{2}{|c|}{$30-50 \mathrm{eV}$} & \multicolumn{2}{|c|}{$50-70 \mathrm{eV}$} \\
\hline & $\mathrm{C} 1 \mathrm{~s}-\pi *$ & $\mathrm{O} 1 \mathrm{~s}-\pi^{*}$ & $\mathrm{C} 1 \mathrm{~s}-\pi^{*}$ & $\mathrm{O} 1 \mathrm{~s}-\pi^{*}$ \\
\hline $\mathrm{C}^{+}$ & 289 & 262 & 382 & 329 \\
\hline $\mathrm{O}^{+}$ & 195 & 223 & 363 & 291 \\
\hline $\mathrm{CO}_{2}{ }^{2+}$ & 1.3 & 2 & 7.0 & 3.9 \\
\hline $\mathrm{CO}^{+}$ & 507 & 511 & 240 & 372 \\
\hline $\mathrm{O}_{2}^{+}$ & 7.3 & 2.3 & 6.3 & 3.3 \\
\hline
\end{tabular}

${ }^{a}$ In single ionization, relative to the ground state of neutral $\mathrm{CO}_{2}$. The energy content of doubly charged products is not defined.
$\mathrm{O}_{2}{ }^{+}$intensity remains. From this we conclude, in agreement with Laksman et al., that the origin of this effect is primarily neither a difference in population of specific ionized states accessed by Auger decays, nor a property of ionized states in a restricted range of internal energy. Instead, the essential process must happen in the initial excited state. There must be a region of the coordinate space, outside the normal FranckCondon zone, which is reached only by nuclear motion in the core-excited state and from which all forms of Auger decay populate regions of potential energy surfaces leading to $\mathrm{O}_{2}{ }^{+}$.

The third question to be considered is the origin of the relatively higher yield of $\mathrm{CO}_{2}{ }^{2+}$ in ionization at the $\mathrm{O} \mathrm{K}$ line than at the $\mathrm{C} \mathrm{K}$-line. The difference in the Auger spectra is clearly known ${ }^{28}$ but it remains to be seen whether this alone is the vital factor, or whether, within the internal energy range where this ion is formed, for the same total energy deposited, one ionization route is more effective than the other. Double photoionization of $\mathrm{CO}_{2}$ populates effectively stable bound states of the doubly charged ion from $37.3 \mathrm{eV}$ to $39.2 \mathrm{eV}$ (onset of $\mathrm{O}^{+}+\mathrm{CO}^{+5}$ ) and metastable states showing vibrational structure up to at least $47 \mathrm{eV} .^{9,36}$ Within the range of energy just above the double ionization limit, the mass spectrum varies strongly, changing from pure $\mathrm{CO}_{2}{ }^{2+}$ to $\mathrm{O}^{+}+\mathrm{CO}^{+}$with some remaining $\mathrm{CO}_{2}{ }^{2+}$ and later to $\mathrm{C}^{+}$with other products. To test whether the branching in dissociation is a function of energy only, it would be necessary to compare mass spectra from population of the same narrow band of energies, just above or around $39 \mathrm{eV}$ by Auger decay from the two different holes. Unfortunately, the electron energy resolution in the present setup $(\sim 15 \mathrm{eV}$ at $250 \mathrm{eV}$ and $30 \mathrm{eV}$ at $500 \mathrm{eV})$ is insufficient for this task.

\section{DISCUSSION AND CONCLUSIONS}

The essential factor in formation of $\mathrm{O}_{2}{ }^{+}$by ionization of $\mathrm{CO}_{2}$ is shown to be bending towards the requisite molecular configuration in the core-excited state. The lifetimes of a few fs $\left(\sim 6 \mathrm{fs}\right.$ for $\mathrm{CK}^{-1}$ and $3.5 \mathrm{fs}$ for $\left.\mathrm{OK}^{-1}\right)$ are short relative to a full period of the bending vibration, which is $50 \mathrm{fs}$ in the neutral molecule, so initialization rather than completion of bending can be envisaged. Since lifetimes are very similar in the excited state and in the core-ionized state where $\mathrm{O}_{2}{ }^{+}$is not produced, the $\pi^{*}$ orbital's bonding character must play a decisive role. The very weak formation of $\mathrm{O}_{2}{ }^{+}$in valence single ionization (cf. Fig. 2) may be attributable to partial population of this orbital in inner-valence states. The thermodynamic limit for forming the ion pair $\mathrm{C}^{+}+\mathrm{O}_{2}{ }^{+}$with zero kinetic energy is $34.8 \mathrm{eV}$, while for formation of $\mathrm{C}+\mathrm{O}_{2}{ }^{+}$it is $23.5 \mathrm{eV}$. The present low resolution and some uncertainty in the energy calibration make it difficult to deduce an experimental onset energy from Fig. 3 or Fig. 4, but our best estimate (from comparison with the onsets of $\mathrm{CO}_{2}{ }^{+}$and $\mathrm{CO}_{2}{ }^{2+}$ ) is in the region of $32 \mathrm{eV}$. If the $\mathrm{C}^{+}+\mathrm{O}_{2}{ }^{+}$ion pair is born with $6 \mathrm{eV}$ kinetic energy release, which is the most probable energy from Laksman et al. (cf. their Fig. 2), this pair cannot appear below $40.8 \mathrm{eV}$ internal energy. Thus, the crude experimental onset again points to an origin partly in single ionization, but one which is well above the thermodynamic limit. This means 
that as singly ionized products, the fragments must either be in excited internal states or have considerable $(\sim 8 \mathrm{eV})$ kinetic energy. From the observation of $\mathrm{O}_{2}{ }^{+}$with high kinetic energy in single ionization by resonant Auger decay from the C1s$\pi^{*}$ resonance we surmised above that capture of a shaken-up electron might be involved. Such capture by a $\mathrm{C}^{+}$ion would probably leave the $\mathrm{C}$ atom in an excited state, so this explanation is compatible with high internal energy in the products. Furthermore, even in a bent form of $\mathrm{CO}_{2}$, the $\mathrm{O}-\mathrm{O}$ distance is very unlikely to be the same as in $\mathrm{O}_{2}{ }^{+}$, giving a further reason for internal excitation.

On the reason for the different relative intensities of the $\mathrm{O}_{2}{ }^{+}$ion from the carbon and oxygen pre-edge resonances, our results lead us to concur with the view that the essential step is strong bending of the molecule in the neutral excited state before Auger decay. The subsequent Auger decay then retains population in parts of the coordinate space inaccessible by vertical transitions from the ground state, which lead to the symmetric products $\mathrm{C}^{+}+\mathrm{O}_{2}{ }^{+}$. This seems compatible with the observation of $\mathrm{S}_{2}{ }^{+}$from normal ionizing transitions in $\mathrm{CS}_{2}$, but not in the more rigid OCS or $\mathrm{CO}_{2}$. To test this idea it would be interesting to try a pump-probe experiment where the bending mode of $\mathrm{CO}_{2}$ is highly excited before ionization.

While we cannot pin down the reason for the site-specific effect on the abundance of $\mathrm{CO}_{2}{ }^{2+}$, we have demonstrated the case and hope for a conclusive test in the future. It is, of course, unsurprising that a triatomic molecule reacts in specific ways in distinct quantum states, since internal energy flow is impeded by the low vibronic state density. But whether or not a very high electronic state density, as in multiply charged ions, can compensate for this, remains an open question.

\section{ACKNOWLEDGMENTS}

This work has been financially supported by the Swedish Research Council (VR), the Göran Gustafsson Foundation (UU/KTH), the Carl Trygger Foundation, and the Knut and Alice Wallenberg Foundation, Sweden. We would like to warmly acknowledge the support by the staff and colleagues at the Helmholtz Centre for Materials and Energy $\mathrm{GmbH}$ BESSY-II, Berlin. This work was also supported by the European Community - Research Infrastructure Action under the FP6 "Structuring the European Research Area" Programme (through the Integrated Infrastructure Initiative "Integrating Activity on Synchrotron and Free Electron Laser Science" Contract No. R II 3-CT-2004-506008).

${ }^{1}$ N. Bussières and P. Marmet, Can. J. Phys. 55, 1889 (1977).

${ }^{2}$ J. Berkowitz and J. H. D. Eland, J. Chem. Phys. 67, 2740 (1977).
${ }^{3}$ T. Masuoka and J. A. R. Samson, Journal de Chimie Physique 77, 623 (1980).

${ }^{4}$ R. Bombach, J. Dannacher, J.-P. Stadelman, and J. C. Lorquet, J. Chem. Phys. 79, 4214 (1983).

${ }^{5}$ T. Masuoka, Phys. Rev. A 50, 3886 (1994).

${ }^{6}$ R. Locht, Int. J. Mass Spectrom. Ion Processes 148, L17 (1995).

${ }^{7}$ G. Öhrwall, M. M. Sant'Anna, W. C. Stolte, I. Dominguez-Lopez, L. T. N. Dang, A. S. Schlachter, and D. W. Lindle, J. Phys. B: At. Mol. Opt. Phys. 35, 4543 (2002).

${ }^{8}$ J. Liu, W. Chen, M. Hochlaf, X. Qian, C. Chang, and C. Y. Ng, J. Chem. Phys. 118, 149 (2003).

${ }^{9}$ A. E. Slattery, T. A. Field, M. Ahmad, R. I. Hall, J. Lambourne, F. Penent, P. Lablanquie, and J. H. D. Eland, J. Chem. Phys. 122, 084317 (2005).

${ }^{10}$ Z. D. Pešić, D. Rolles, R. C. Bilodeau, I. Dimitriu, and N. Berrah, Phys. Rev. A 78, 051401 (2008).

${ }^{11}$ Y. Muramatsu, K. Ueda, N. Saito, H. Chiba, M. Lavollée, A. Czasch, T. Weber, O. Jagutzki, H. Schmidt-Böcking, R. Moshammer, U. Becker, K. Kubozuka, and I. Koyano, Phys. Rev. Lett. 88, 133002 (2002).

${ }^{12}$ R. K. Kushawaha, S. S. Kumar, I. A. Prajapati, K. P. Subramanian, and B. Bapat, J. Phys. B: At. Mol. Opt. Phys. 42, 105201 (2009).

${ }^{13}$ J. Laksman, E. P. Månsson, C. Grunewald, A. Sankari, M. Gisselbrecht, D. Céolin, and S. L. Sorensen, J. Chem. Phys. 136, 104303 (2012).

${ }^{14}$ D. Ceolin, C. Miron, M. Simon, and P. Morin, J. Electron Spectrosc. Relat. Phenom. 141, 171 (2004).

${ }^{15}$ J. Adachi, N. Kosugi, E. Shigemasa, and A. Yagishita, J. Chem. Phys. 107, 4919 (1997).

${ }^{16}$ P. Morin, M. Simon, C. Miron, N. Leclercq, E. Kukk, J. D. Bozek, and N. Berrah, Phys. Rev. A 61, 050701 (2000).

${ }^{17}$ T. Masuoka, J. Electron Spectrosc. Relat. Phenom. 101, 53 (1999).

${ }^{18}$ J. D. Bozek, N. Saito, and I. H. Suzuki, Phys. Rev. A 51, 4563 (1995).

${ }^{19}$ M. Alagia, P. Candori, S. Falcinelli, M. Lavollée, F. Pirani, R. Richter, S. Stranges, and F. Vecchiocattivi, J. Phys. Chem. A 113, 14755 (2009).

${ }^{20}$ N. Neumann, D. Hant, L. Ph, H. Schmidt, J. Titze, T. Jahnke, A. Czasch, M. S. Schöffler, K. Kreidi, O. Jagutzki, H. Schmidt-Böcking, and R. Dörner, Phys. Rev. Lett. 104, 103201 (2010).

${ }^{21}$ P. Roy, I. Nenner, P. Millie, P. Morin, and D. Roy, J. Chem. Phys. 84, 2050 (1986).

${ }^{22}$ H. Hogreve, J. Phys. B: At. Mol. Opt. Phys. 28, L263 (1995).

${ }^{23}$ M. Hochlaf, F. R. Bennett, G. Chambaud, and P. Rosmus, J. Phys. B: At. Mol. Opt. Phys. 31, 2163 (1998).

${ }^{24}$ P. Millié, I. Nenner, P. Archirel, P. Lablanquie, P. Fournier, and J. H. D. Eland, J. Chem. Phys. 84, 1259 (1986).

${ }^{25}$ J. H. D. Eland, L. Andric, P. Linusson, L. Hedin, S. Plogmaker, J. Palaudoux, F. Penent, P. Lablanquie, and R. Feifel, J. Chem. Phys. 135, 134309 (2011).

${ }^{26}$ D. Aitchison and J. H. D. Eland, Chem. Phys. 263, 449 (2001).

${ }^{27}$ D. Mathur and F. M. Harris, Mass Spectrom. Rev. 8, 269 (1989).

${ }^{28}$ V. Brites, J. H. D. Eland, and M. Hochlaf, Chem. Phys. 346, 23 (2008).

${ }^{29}$ Y. Shibata, K. Soejima, H. Iwayama, E. Shigemasa, and Y. Hikosaka, UVSOR Annual Activity Report (2012), ISSN 0911-5730, p. 59.

${ }^{30}$ J. H. D. Eland and R. Feifel, Chem. Phys. 327, 85 (2006).

${ }^{31}$ R. Feifel, J. H. D. Eland, L. Storchi, and F. Tarantelli, J. Chem. Phys. 125, 194318 (2006)

${ }^{32}$ S. Plogmaker, P. Linusson, J. H. D. Eland, N. Baker, E. M. J. Johansson, H. Rensmo, R. Feifel, and H. Siegbahn, Rev. Sci. Instrum. 83, 013115 (2012).

${ }^{33}$ J. H. D. Eland, O. Vieuxmaire, T. Kinugawa, P. Lablanquie, R. I. Hall, and F. Penent, Phys. Rev. Lett. 90, 053003 (2003).

${ }^{34}$ W. Eberhardt, J.-E. Rubensson, K. J. Randall, J. Feldhaus, A. L. D. Kilcoyne, A. M. Bradshaw, Z. Xu, P. D. Johnson, and Y. Ma, Phys. Scr. T41, 143 (1992).

${ }^{35}$ S. Sundin, S. J. Osborne, A. Ausmees, O. Björneholm, S. L. Sorensen, A. Kikas, and S. Svensson, Phys. Rev. A 56, 480 (1997).

${ }^{36}$ J. H. D. Eland, Chem. Phys. 294, 171 (2003). 